\title{
Parabiosis and single-cell RNA sequencing reveal a limited contribution of monocytes to myofibroblasts in kidney fibrosis
}

\author{
Rafael Kramann, ${ }^{1}$ Flavia Machado, ${ }^{2}$ Haojia Wu, ${ }^{2}$ Tetsuro Kusaba, ${ }^{3}$ Konrad Hoeft, ${ }^{1}$ \\ Rebekka K. Schneider, ${ }^{4,5}$ and Benjamin D. Humphreys ${ }^{2}$ \\ 'Division of Nephrology and Clinical Immunology, RWTH Aachen University, Aachen, Germany. \\ ${ }^{2}$ Division of Nephrology, Department of Medicine and Department of Cell Biology, Washington University School of \\ Medicine, St. Louis, Missouri, USA. ${ }^{3}$ Department of Nephrology, Graduate School of Medical Science, Kyoto Prefectural \\ University of Medicine, Kyoto, Japan. ${ }^{4}$ Department of Hematology, Erasmus MC Cancer Institute, Rotterdam, Netherlands. \\ ${ }^{5}$ Division of Hematology, RWTH Aachen University, Aachen, Germany.
}

\begin{abstract}
Fibrosis is the common final pathway of virtually all chronic injury to the kidney. While it is well accepted that myofibroblasts are the scar-producing cells in the kidney, their cellular origin is still hotly debated. The relative contribution of proximal tubular epithelium and circulating cells, including mesenchymal stem cells, macrophages, and fibrocytes, to the myofibroblast pool remains highly controversial. Using inducible genetic fate tracing of proximal tubular epithelium, we confirm that the proximal tubule does not contribute to the myofibroblast pool. However, in parabiosis models in which one parabiont is genetically labeled and the other is unlabeled and undergoes kidney fibrosis, we demonstrate that a small fraction of genetically labeled renal myofibroblasts derive from the circulation. Single-cell RNA sequencing confirms this finding but indicates that these cells are circulating monocytes, express few extracellular matrix or other myofibroblast genes, and express many proinflammatory cytokines. We conclude that this small circulating myofibroblast progenitor population contributes to renal fibrosis by paracrine rather than direct mechanisms.
\end{abstract}

Conflict of interest: The authors have declared that no conflict of interest exists.

Submitted: January 2, 2018

Accepted: April 4, 2018

Published: May 3, 2018

Reference information: JCI Insight. 2018;3(9):e99561. https:// doi.org/10.1172/jici.insight.99561.

\section{Introduction}

Chronic kidney disease (CKD) affects $10 \%$ of the population of Europe and the United States, and disease prevalence is increasing steadily. Renal interstitial fibrosis correlates well with kidney functional decline, and inhibition of fibrosis is a promising strategy to slow down progression of CKD. While it is well accepted that myofibroblasts are the cells that cause fibrosis, the cellular origin of kidney myofibroblasts is still very controversial (1-3). We have reported that approximately half of all kidney myofibroblasts are derived from the pericyte lineage (4). However, the origins of the other half remains unclear. A precise definition of the cellular origins of kidney myofibroblasts represents a key step to understanding fibrosis and developing therapeutics to slow progression of $\mathrm{CKD}$.

The contribution of proximal tubular epithelium to the myofibroblast pool via epithelial-to-mesenchymal transition has been controversial for almost 20 years and is ongoing (5-9). Genetic fate-tracing experiments have come to different conclusions, reporting no contribution (9), a major contribution (10), or a minor contribution of the epithelium (11).

Similarly, the degree to which circulating progenitor cells contribute to the myofibroblast pool has also been debated for many years $(2,12,13)$. Bone marrow-derived mesenchymal stem cells (MSCs) have been reported to contribute to as much as $35 \%$ of the renal myofibroblast pool (11). Furthermore, fibrocytes and macrophages have also been defined as kidney myofibroblast progenitors. Fibrocytes were originally described more than two decades ago as a leukocyte-derived population of fibroblast-like cells in subcutaneously implanted wound chambers that express extracellular matrix proteins, such as collagen I (14). Regarding a contribution of fibrocytes to the myofibroblast pool in kidney fibrosis, studies from different groups have led to different conclusions. While bone marrow transplantation experiments using collagen 1a1 or collagen $1 \mathrm{a} 2$ reporter chimeras suggest no significant contribution of circulating bone 
marrow-derived cells to the myofibroblast pool $(15,16)$, other groups have reported a major contribution of hematopoietic lineage-derived fibrocytes and macrophages to kidney fibrosis (17-24).

Most studies to date have relied heavily on confocal microscopy and/or bone marrow transplantation experiments. Discriminating myofibroblasts from hematopoietic lineage cells by confocal microscopy is technically challenging due to their thin, branched architecture and to their abundance in the renal interstitium after injury. Furthermore, bone marrow transplantation experiments using genetic reporters result in engraftment solely of hematopoietic stem cells, whereas other bone marrow cells, such as MSCs, do not engraft well $(4,25)$, precluding analysis of the contribution of MSC lineage cells to the myofibroblast pool. Using an inducible genetic fate-tracing model, we confirmed that proximal tubular epithelium does not contribute to the myofibroblast pool. In order to dissect the contribution of any circulating cells to the renal myofibroblast pool, we have performed parabiosis and single-cell RNA sequencing (scRNA-seq) experiments. Our data indicate that monocytes contribute a small fraction of myofibroblasts in kidney fibrosis, whereas most myofibroblasts are derived from resident mesenchymal cells, such as pericytes and resident fibroblasts.

\section{Results}

Inducible genetic fate tracing indicates no contribution of proximal tubular epithelium to the myofibroblast pool in kidney fibrosis. We recently developed an inducible and proximal tubule-specific Cre driver (26) by knocking a CreER ${ }^{\mathrm{t} 2}$ cassette into the sodium-dependent inorganic phosphate transporter SLC34a1 locus, which is expressed only in the proximal tubule. In order to perform inducible genetic fate tracing of proximal tubular epithelium, we generated bigenic SLC34a1-GFPCreER ${ }^{\mathrm{t} 2}$; tdTomato mice (Figure 1A). Eight-week-old mice were pulsed with high-dose tamoxifen and subjected to unilateral ureteral obstruction (UUO) surgery at 10 days after the last tamoxifen dose (Figure 1B). Careful evaluation and quantification of kidney sections costained for $\alpha$-SMA indicated that proximal tubular epithelial cells do not become interstitial myofibroblasts (Figure 1, C and D)

Parabiosis model with fate tracing of all cells from one mouse and kidney fibrosis induction in the other. To quantitate and better describe the contribution of circulating cells to the kidney myofibroblast pool, we performed parabiosis experiments with generalized genetic cell fate tracing in one parabiont and induction of kidney fibrosis in the other. To ubiquitously genetically label cells with the bright red fluorochrome tdTomato, bigenic Rosa26CreER;tdTomato mice received tamoxifen and underwent parabiosis surgery at 10 days after the last tamoxifen dose (Figure 2A). The Rosa26CreER;tdTomato mice were conjoined with B6-CD 45.1 $1^{+}$mice, which do not express tdTomato but express a different isoform of the pan-leukocyte marker CD45, which can be discriminated by flow cytometry (B6-CD45. $1^{+}$, as opposed to Rosa26CreER;tdTomato-CD45.2+) (Figure 2A). Shared circulation and recombination efficiency were verified 4 weeks after parabiosis surgery and before induction of kidney fibrosis (Figure 2, B and C). The analysis showed a good cross-circulation, indicated by an almost $1: 1$ ratio of CD $45.1^{+}$and CD $45.2^{+}$cells and a recombination efficiency of $>90 \%$ (Figure 2, B and C). The B6-CD45.1 parabiont was then subjected to UUO surgery to assess whether any circulating tdTomato ${ }^{+}$cells from the Rosa26CreER;tdTomato $\left(\mathrm{CD} 45.2^{+}\right)$parabiont would contribute to the myofibroblast pool during kidney fibrosis. Mice were sacrificed 10 days after UUO surgery. The contralateral noninjured kidney (CLK) served as an internal control. Development of fibrosis in the UUO model was confirmed by trichrome staining and quantification (Supplemental Figure 1, A and B; supplemental material available online with this article; https://doi. org/10.1172/jci.insight.99561DS1). Flow cytometric evaluation of PBS-perfused spleens from CD45.1 mice showed a cross-circulation with $\mathrm{CD} 45.1^{+}$and CD $45.2^{+}$leukocytes from both mice and confirmed efficient recombination (Figure 2, D and E). As expected, UUO surgery resulted in a tremendous influx of leukocytes into the UUO kidneys. More than half of the leukocytes were derived from the CD45.2 (Rosa26CreER;tdTomato) parabiont (Figure 2, F-H), confirming the effectiveness of the cross-circulation and an optimal experimental set up to study influx of circulating cells from the conjoined mouse. Representative gating on living, single kidney cells is shown in Supplemental Figure 1C.

Circulating cells contribute to a minor fraction of renal myofibroblasts. We next measured the contribution of circulating cells (tdTomato ${ }^{+}$) to the myofibroblast lineage. Half of the fibrotic (UUO) and control (CLK) kidneys were subjected to flow cytometric evaluation and costaining with the myofibroblast marker $\alpha$-smooth muscle actin ( $\alpha$-SMA). Flow cytometric evaluation after gating on single and living (DAPI ${ }^{-}$) cells indicated that, indeed, a small fraction of renal myofibroblasts was derived from circulation (tdTomato ${ }^{+} / \alpha-$ 
A

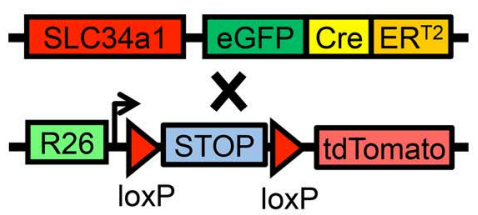

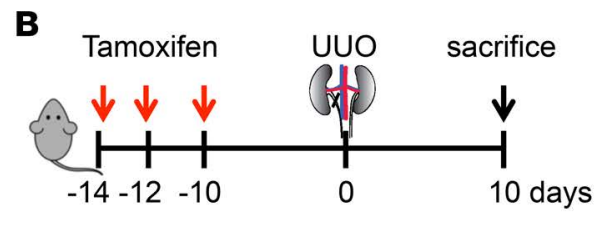

\section{c}
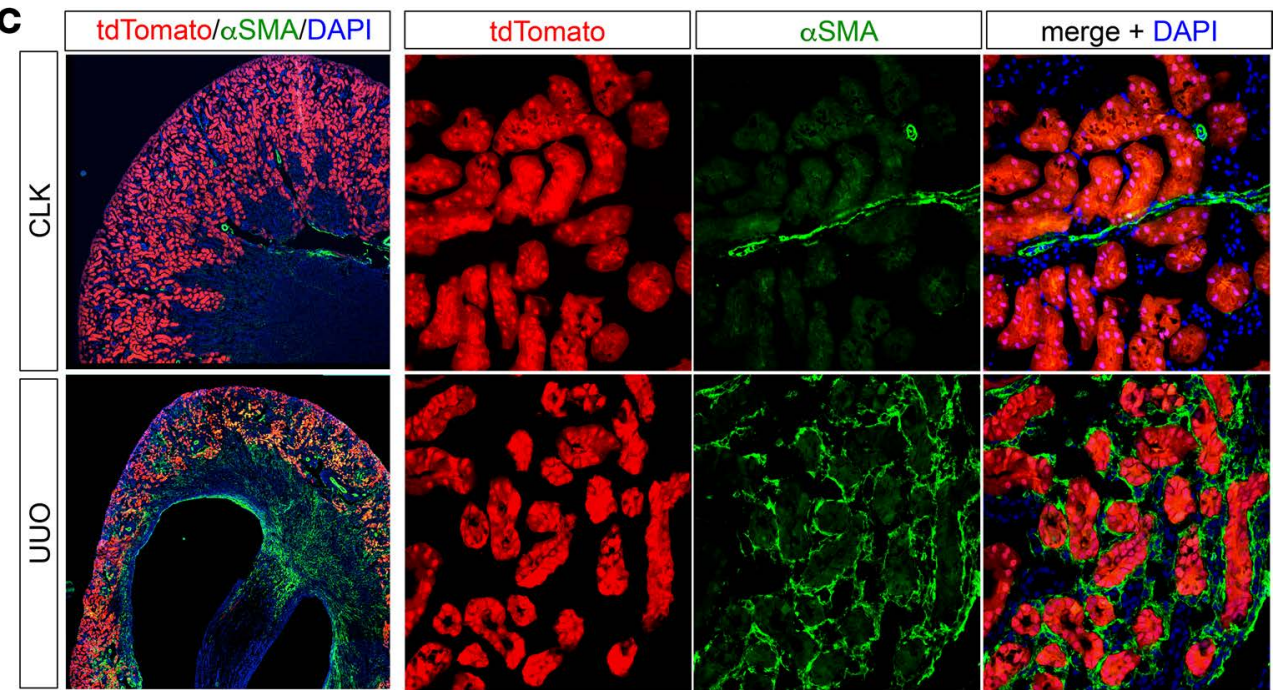

D

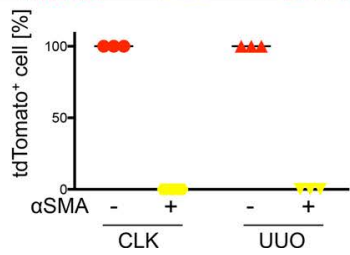

Figure 1. Inducible genetic fate tracing indicates no contribution of proximal tubular epithelium to kidney myofibroblasts. (A) Scheme of the generation of SCL34a1GFPCreER ${ }^{\mathrm{t} 2}$; tdTomato mice. (B) Scheme of the genetic fate-tracing experiment; 8-week-old SLC34a1CFPCreER;tdTomato mice ( $n=3$ males) were pulsed with tamoxifen $(3 \times 10 \mathrm{mg}$ p.o.) and subjected to UUO surgery at 10 days after the last tamoxifen dose. Mice were sacrificed 10 days after surgery. (C) Representative images of contralateral noninjured (CLK) and injured (unilateral ureteral obstruction [UUO]) kidneys stained for $\alpha$-SMA. Original magnification, $\times 4$ (first and third columns); $\times 60$ (second and fourth columns). (D) Quantification of tdTomato ${ }^{+}$and $\alpha-S M A^{+}$versus $\alpha-S M A^{-}$cells. All data represent mean \pm SD.

$\mathrm{SMA}^{+}$) (Figure 3, A-F). While we observed a tremendous increase of both tdTomato ${ }^{+}$cells (influx of all circulating cells) and $\alpha-\mathrm{SMA}^{+}$cells (expansion of myofibroblasts) after UUO, as expected, only a small fraction of myofibroblasts coexpressed tdTomato, indicating that they originated from the Rosa26CreER;tdTomato parabiont (Figure 3, A-F). Confocal microscopy of the Rosa26CreER;tdTomato (CD45.2) kidney confirmed the recombination efficiency and showed that all cells were tdTomato ${ }^{+}$(Figure $3 \mathrm{G}$ ).

To confirm the flow cytometric data, we used high-resolution confocal microscopy (Airyscan detector) to localize tdTomato ${ }^{+}$myofibroblasts that were derived from circulation. While we only observed sparse tdTomato $^{+}$cells in the CLK kidneys of the B6-CD45.1 parabiont, high-resolution confocal microscopy confirmed areas with large numbers of tdTomato ${ }^{+}$cells in the UUO kidneys. Importantly, we observed

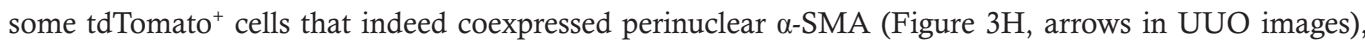
thus confirming the flow cytometric data with high-resolution imaging of renal interstitium.

Flow cytometric analysis revealed that all cells in the UUO kidneys that were derived from circulation (tdTomato ${ }^{+}$) also coexpressed CD45.2 (Figure 4, A and B) and, thus, were of hematopoietic lineage. Indeed, costaining for CD45 with subsequent confocal microscopy showed that tdTomato ${ }^{+}$cells in the kidneys showed a thin layer of CD45 membrane staining, confirming their hematopoietic origin (Figure 4, $\mathrm{C}$ and $\mathrm{D}$, insets). We next aimed to identify a combination of surface markers that would allow isolation of myofibroblasts from circulation in comparison to noncirculating myofibroblasts. As we have previously reported that all renal myofibroblasts coexpress the mesenchymal marker PDGFR $\beta$ (4) and PDGFR $\beta$ has also been described as a marker of circulating fibrocytes (27), a combination of PDGFR $\beta$ with CD45 


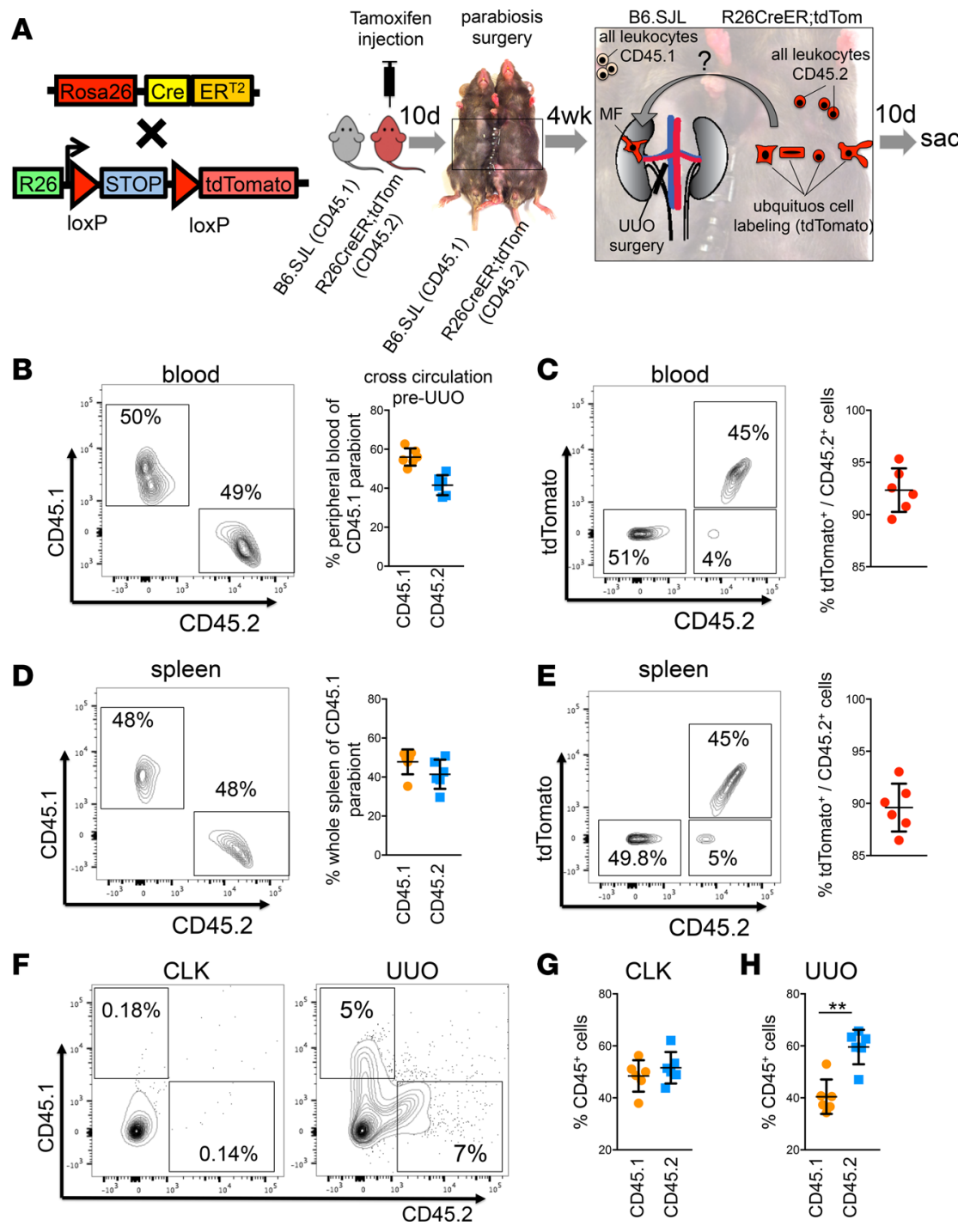

Figure 2. Parabiosis with genetic fate tracing to dissect the contribution of circulating cells to kidney fibrosis. (A) Rosa26CreER; tdTomato mice ( $n=$ 8; all females, 8 week of age) received tamoxifen ( $4 \times 10 \mathrm{mg}$ p.o. every other day) to genetically tag all cells and were conjoined with B6.SJL (CD45.1) mice at 10 days after the last tamoxifen dose. Four weeks after parabiosis surgery the B6.SJL parabiont was subjected to unilateral ureteral obstruction (UUO) surgery to induce kidney fibrosis. Mice were sacrificed 10 days after UUO surgery. $n=2$ mice died during the experiment; final data represent $n=6$ parabiosis pairs in all readouts. MF, myofibroblast. (B) Representative flow cytometric plot and quantification of CD45.1 versus CD45.2 $2^{+}$ cells in the blood of the B6.5JL (CD45.1) parabiont at 4 weeks after parabiosis surgery. (C) Representative flow cytometric plot and quantification of recombination efficiency (i.e., tdTomato+) of CD45.2+ cells in the blood of the B6.SJL parabiont at 4 weeks after parabiosis surgery. (D) Representative flow cytometric plots and quantification of CD $45.1^{+}$versus CD $45.2^{+}$cells in the spleen of the B6.SJL (CD45.1) parabiont after sacrifice. (E) Representative flow cytometric plot and quantification of recombination efficiency (i.e., tdTomato ${ }^{+}$) of CD45.2+ cells in the spleen of the B6.SJL parabiont after sacrifice. (F-H) Representative flow cytometric plots and quantification of CD45.1 $1^{+}$versus CD45. $2^{+}$leukocyte influx into the contralateral noninjured (CLK) and fibrotic UUO kidneys. All data represent mean $\pm \mathrm{SD} .{ }^{*} P<0.01$ by unpaired $t$ test $n=6$ in each graph.

would allow the isolation of circulating hematopoietic myofibroblasts $\left(\mathrm{PDGFR} \beta^{+} \mathrm{CD} 45^{+}\right.$) and resident nonhematopoietic (PDGFR $\beta^{+} \mathrm{CD} 45^{-}$) cells. Indeed, costaining of kidneys for PDGFR $\beta$ indicated that the $\mathrm{tdTomato}^{+} / \alpha-\mathrm{SMA}^{+}$population of myofibroblasts derived from circulation coexpressed PDGFR $\beta$ (Figure $3 \mathrm{H})$. Thus, our data suggest that costaining for the pan-leukocyte marker CD45 and the mesenchymal marker PDGFR $\beta$ can be used to isolate both resident and circulating kidney myofibroblasts.

scRNA-seq confirms a contribution of circulating $C D 45^{+}$cells to the myofibroblast pool. We next turned to an unbiased nonparabiosis approach to confirm our genetic fate-tracing parabiosis results with scRNAseq from FACS isolated PDGFR $\beta^{+} \mathrm{CD} 45^{+}$and PDGFR $\beta^{+} \mathrm{CD} 45^{-}$cells. C57BL/ 6 wild-type mice were subjected to UUO surgery and sacrificed 10 days after surgery. We sorted either myofibroblasts that were derived from circulation $\left(\mathrm{PDGFR} \beta^{+} \mathrm{CD} 45^{+}\right.$) or all other myofibroblasts (PDGFR $\beta^{+} \mathrm{CD} 45^{-}$) as singlets into individual wells of 96-well plates (Supplemental Figure 2A). We sorted two 96-well plates of each population from whole digested UUO kidney samples and subjected the samples to scRNA-seq. The kidney samples were pooled from 3 different mice. Cells from 2 different plates were distributed evenly in the tSNE analysis clusters, excluding batch effects (Supplemental Figure 2B). The tSNE analysis resulted in 2 distinct clusters that were almost identical; the 2 sorted cell populations were made up of PDGFR $\beta^{+} \mathrm{CD} 45^{+}$and PDGFR $\beta^{+} \mathrm{CD} 45^{-}$cells (Figure 5A). The top 30 upregulated genes in both clusters are outlined in Supplemental Figure 2C. Importantly, the scRNA-seq data confirmed the results of the parabiosis fate-tracing experiment and demonstrated that the great majority of myofibroblasts identified by expression of $\alpha$-SMA (Acta2) and or collagen (Colla1, Col3a1) were among the PDGFR $\beta^{+}$CD $45^{-}$ population or resident kidney cells, whereas only a few myofibroblasts were among the population of 

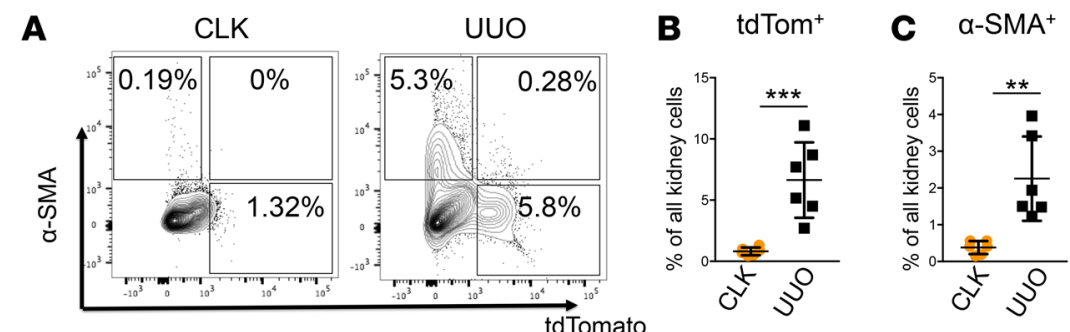

D tdTom ${ }^{+} \alpha-\mathrm{SMA}^{+}$
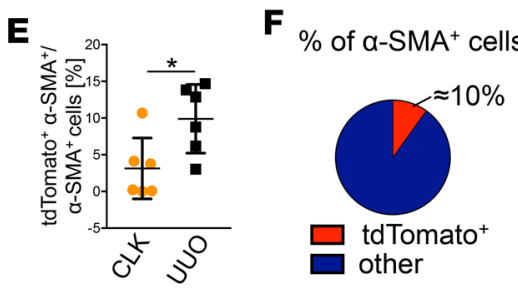

G

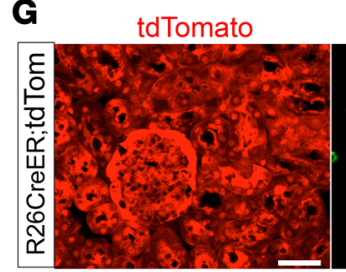

tdTomato

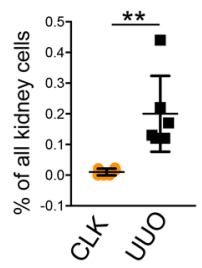

H merge tdTomato / DAP |

a-SMA

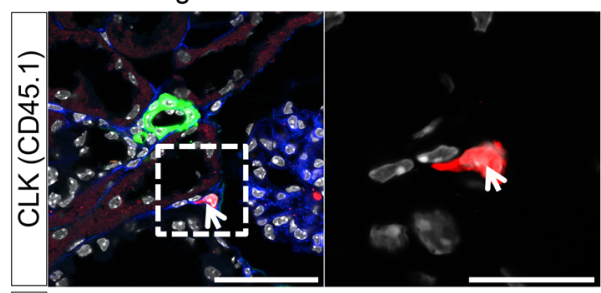

a-SMA / DAPI
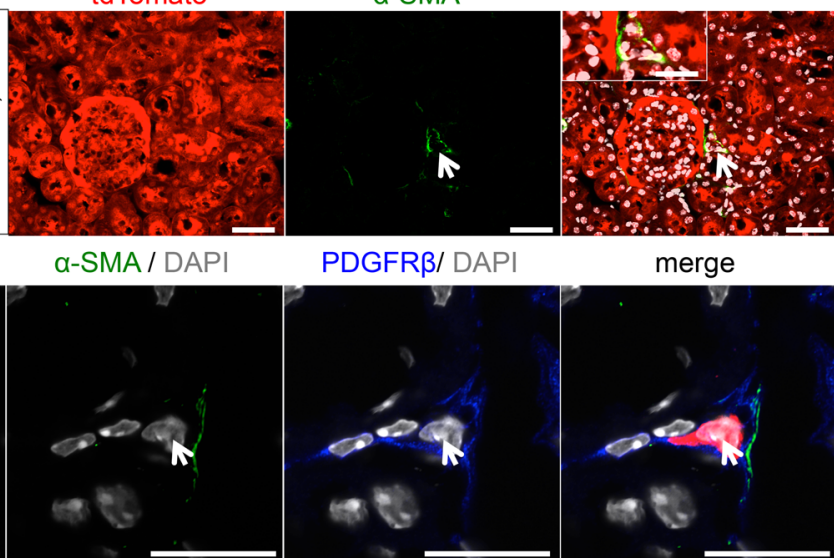

PDGFRß/ DAPI merge
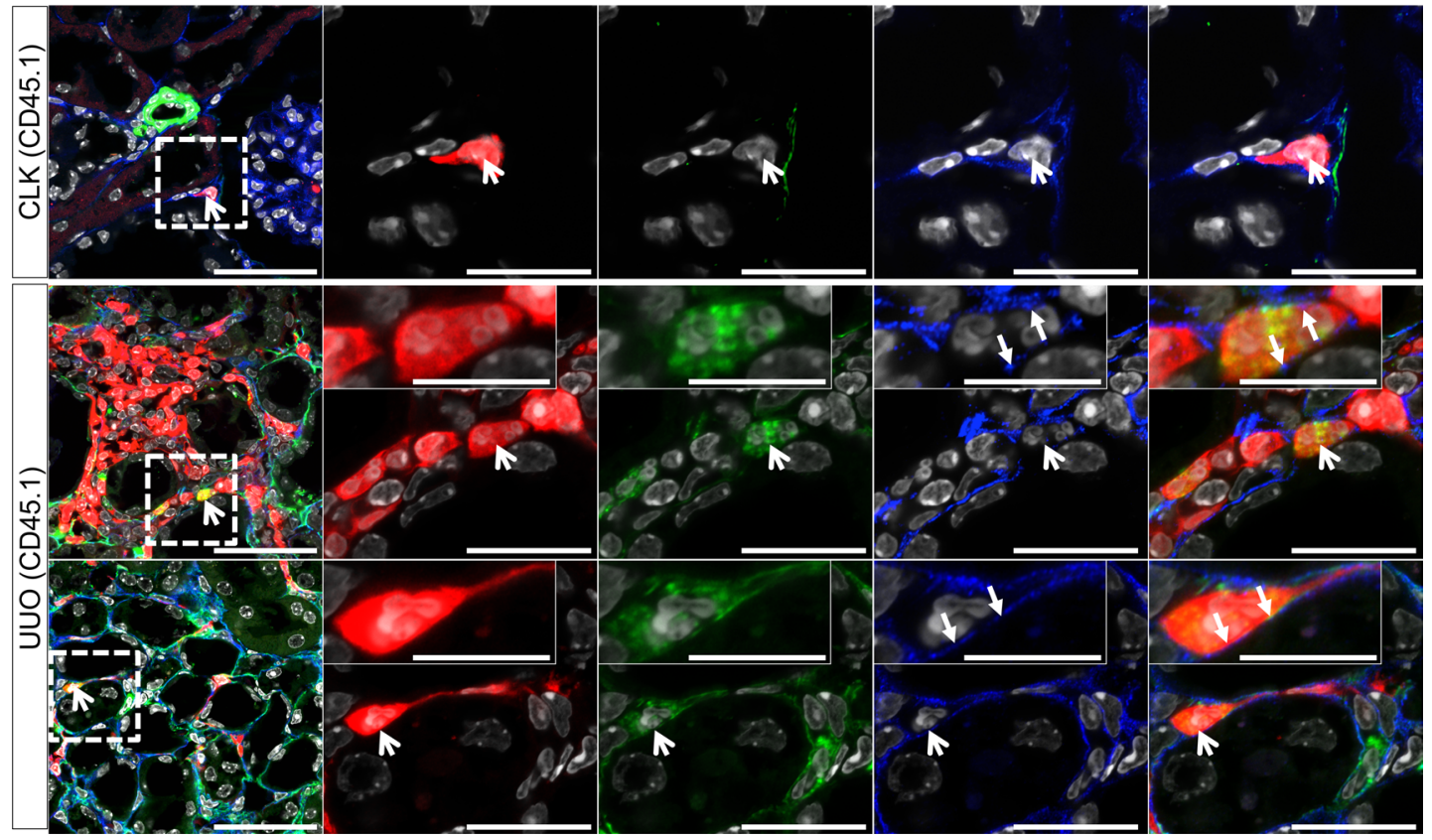

Figure 3. A small fraction of kidney myofibroblasts is derived from circulation. (A) Flow cytometric plots of myofibroblasts ( $\alpha$-SMA+), circulating cells (tdTomato $)$, and $\alpha$-SMA, tdTomato double-positive cells, i.e., myofibroblasts derived from circulation in noninjured contralateral kidneys (CLK) and fibrotic - unilateral ureteral obstruction (UUO) - kidneys from B6.SJL (CD45.1) mice $(n=6)$. (B) Fraction of circulating tdTomato cells from all kidney cells in CLK and fibrotic (UUO) kidneys from B6.SJL (CD45.1) mice $(n=6)$. (C) Fraction of myofibroblasts $\left(\alpha-S M A^{+}\right)$from all kidney cells in CLK and fibrotic (UU0) kidneys from B6.SJL (CD45.1) mice $(n=6)$. (D) Fraction of myofibroblasts derived from circulation ( $\alpha$-SMA ${ }^{+} /$tdTomato $\left.^{+}\right)$from all kidney cells in CLK and fibrotic (UUO) kidneys from B6.SJL (CD45.1) mice $(n=6)$. (E and F) Fraction of myofibroblasts derived from circulation (tdTomato $\left.{ }^{+} / \alpha-S M A^{+}\right)$from all $\alpha-\mathrm{SMA}^{+}$myofibroblasts $(n=6)$. (C) Representative image of the noninjured kidney of the Rosa26CreER;tdTomato parabiont. Scale bars: $50 \mu \mathrm{m} ; 25 \mu \mathrm{m}$ (inset). (H) High-resolution confocal microscopy (Airyscan) images of CLK and fibrotic (UUO) kidneys from B6.SJL (CD45.1) mice costained for $\alpha$-SMA and PDGFR $\beta$. Large arrows indicate tdTomato cells coexpressing $\alpha$-SMA and PDGFR $\beta$. Small arrows indicate the area of thin membranous PDGFR $\beta$ signal. Scale bars: $50 \mu \mathrm{m}$ (column 1); $20 \mu \mathrm{m}$ (columns $2-5$ ); $10 \mu \mathrm{m}$ (insets). All data represent mean \pm SD. ${ }^{*} P<0.05,{ }^{* *} P<0.01,{ }^{* *} P<0.001$ by unpaired $t$ test.

circulating hematopoietic PDGFR $\beta^{+} \mathrm{CD} 45^{+}$cells (Figure 5, B-E). The PDGFR $\beta^{+} \mathrm{CD} 45^{-}$population showed expression of markers that have been associated with pericytes and myofibroblasts, such as Timp1, Col1a1, Col3a1, Rgs5, desmin, CSPG4, and the mesenchymal marker vimentin (Figure 5, B-H, and Supplemental Figure 2C), whereas the population of myofibroblasts derived from circulation (PDG$\mathrm{FR} \beta^{+} \mathrm{CD} 45^{+}$) showed high expression of the monocyte marker CD68 (Figure 5I).

We next examined expression of extracellular matrix proteins as well as chemokines and cytokines that have been associated with fibrosis. Interestingly the resident PDGFR $\beta^{+} \mathrm{CD} 45^{-}$population showed strong expression of various collagens and fibronectin, connective tissue growth factor (CTGF), and TGF- $\beta 3$, whereas the circulating population of $\mathrm{PDGFR} \beta^{+} \mathrm{CD} 45^{+}$cells primarily expressed various 

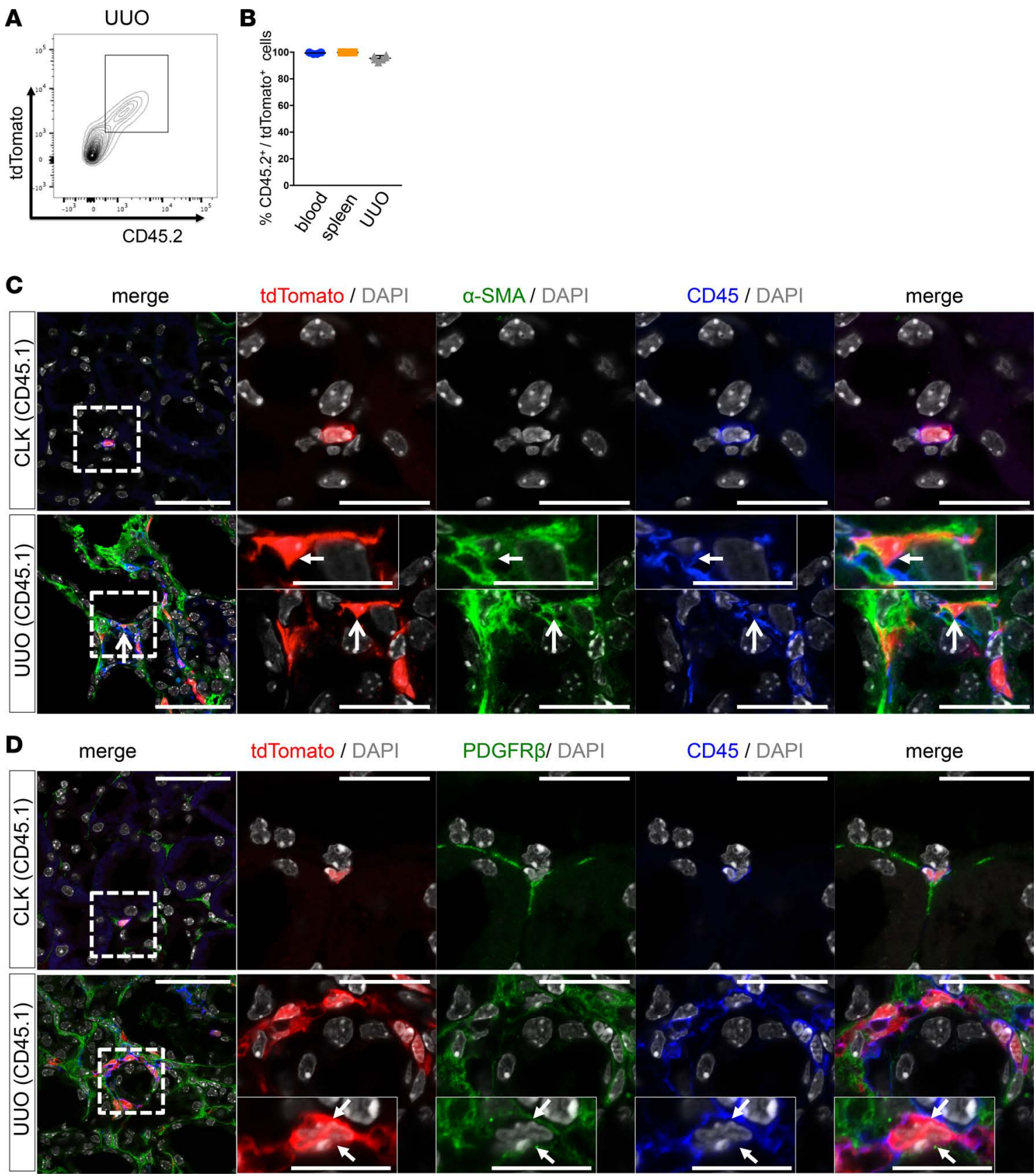

Figure 4. Myofibroblasts derived from circulation coexpress PDGFR $\beta$ and CD45. (A and B) Representative flow cytometric plot and quantification of tdTomato and CD45.2+ cells in blood, spleens, and UUO kidneys of the B6.SJL (CD45.1) mice $(n=6)$. (C) Representative high-resolution confocal microscopy images of contralateral noninjured kidney (CLK) and fibrotic unilateral ureteral obstruction (UUO) kidneys from B6.SJL (CD45.1) mice costained for $\alpha$-SMA and CD45. Large arrows indicate tdTomato cells coexpressing $\alpha$-SMA and CD45. Small arrows indicate the area of thin membranous CD45 signal. Scale bars: $50 \mu \mathrm{m}$ (column 1); $20 \mu \mathrm{m}$ (columns 2-5); $10 \mu \mathrm{m}$ (insets). (D) Representative high-resolution confocal microscopy images of CLK and fibrotic UUO kidneys from B6.SJL (CD45.1) mice costained for PDCFR $\beta$ and CD45. All data represent mean \pm SD; $n=6$. Small arrows indicate the area of thin membranous CD45 and PDGFR $\beta$ signal in the tdTomato cell. Scale bars: $50 \mu \mathrm{m}$ (column 1); $20 \mu \mathrm{m}$ (columns 2-5).

chemokines and interleukins with markedly lower or absent expression of extracellular matrix components compared with the population of resident myofibroblasts (Figure 5J). These data indicate that circulating PDGFR $\beta^{+} \mathrm{CD} 45^{+}$cells only contributed a minor fraction to the myofibroblast pool but might regulate kidney fibrosis in a paracrine fashion by secretion of proinflammatory and profibrotic chemokines. We hypothesized therefore that circulating PDGFR $\beta^{+} C D 45^{+}$cells might interact with resident PDGFR $\beta^{+} C D 45^{-}$cells and looked into receptor-ligand interactions between the 2 cell populations within the scRNA-seq data set. Interestingly, we observed strong expression of multiple receptor-ligand pairs 

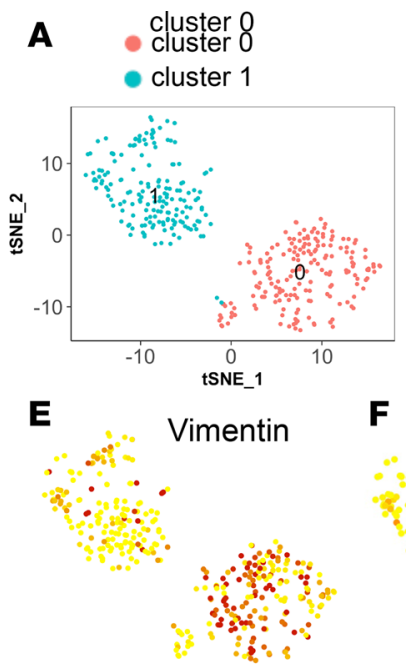

J

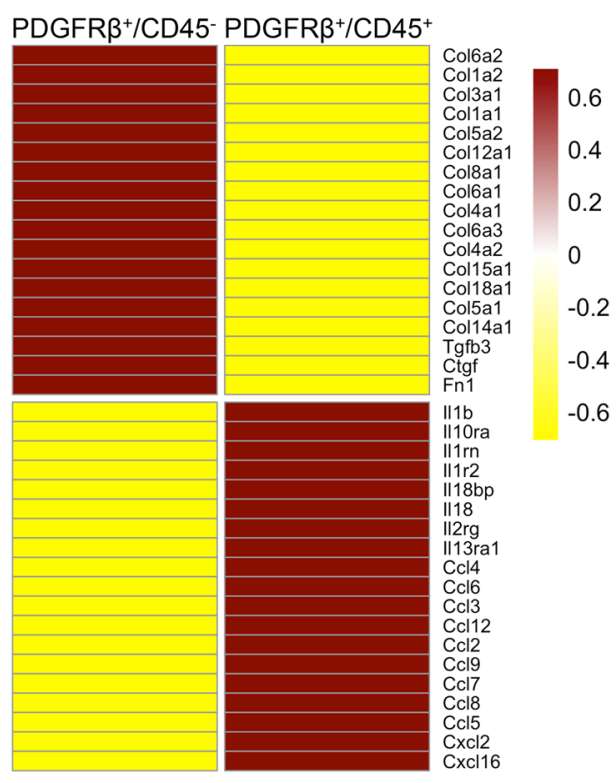

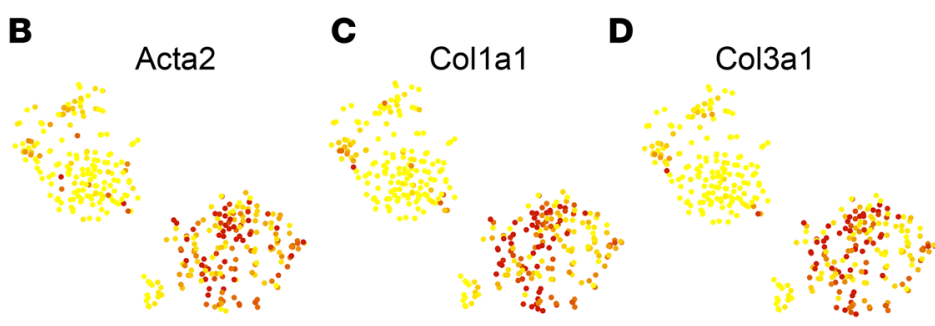

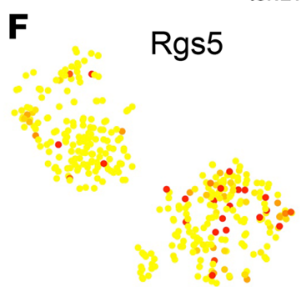

- PDGFR ${ }^{+} / C D 45^{-}$

- $P D G F R \beta^{+} / C D 45^{+}$

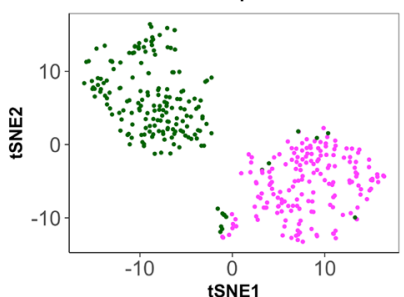

G
H $\quad$ CSPG4

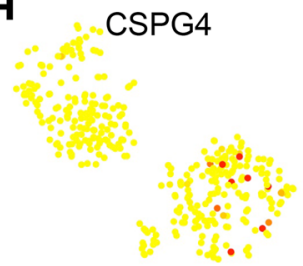

Desmin $\quad \mathbf{H}$

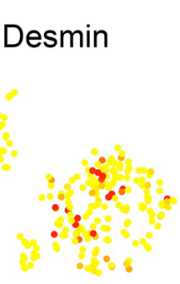

$\mathbf{K}$

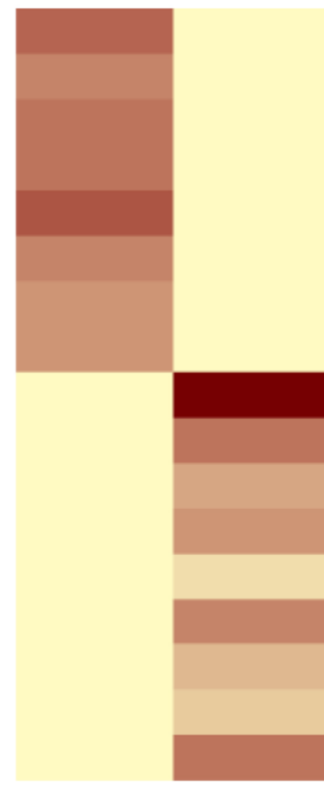

I

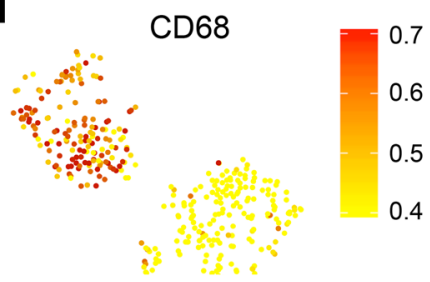

Ligand

PDGFR ${ }^{+} /$CD $^{2} 5^{-}$PDGFR $^{+} / \mathrm{CD}^{2} 5^{+}$

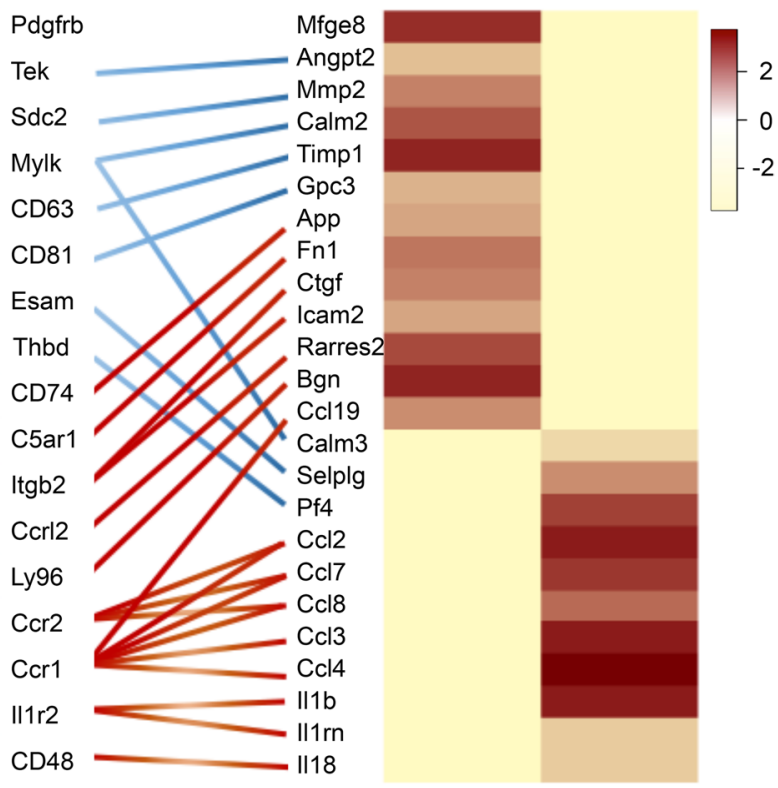

$\mathrm{PDGFR}^{+} / \mathrm{CD}^{2} 5^{-} \mathrm{PDGFR}^{+} / \mathrm{CD} 45^{+}$

Figure 5. Single-cell RNA sequencing reveals that the majority of kidney myofibroblasts is derived from resident pericytes, while a small fraction is derived from circulation. (A) Single-cell RNA sequencing was performed on PDGFR $\beta^{+} C D 45^{-}$cells (myofibroblasts derived from circulation) and PDCFR $\beta^{+}$CD45 $5^{+}$cells (all other myofibroblasts) of whole fibrotic (unilateral ureteral obstruction [UUO]) kidneys. Kidneys were pooled from 3 mice, and two 96-well plates were sorted from each population. tSNE analysis clusters 2 distinct cell populations congruent with the sorted populations. (B-I) Expression levels of genes that define myofibroblasts, pericytes, or macrophages in both tSNE clusters ( $\alpha$-smooth muscle actin [Acta2]; collagen $1 \alpha 1$ [Col1a1]; collagen $3 \alpha 1$ [Col3a1]). Color key denotes the Z-score normalized average expression value of selected differentially expressed genes between PDGFR $\beta^{+} C D 45^{-}$and PDGFR $\beta^{+} C D 45^{+}$cells. (J) Expression levels of extracellular matrix components and proinflammatory, profibrotic cytokines and growth factors between the 2 cell populations. (K) Ligand-receptor pair expression according to cell type. Ligands are indicated on the right, receptors on the left. Lines indicate ligand-receptor pairs. Color key denotes the Z-score normalized average expression value.

between both cell populations (Figure 5K). Among the observed ligands, we found CXCL4/PF4 expression in the circulating cells (PDGFR $\beta^{+} \mathrm{CD} 45^{-}$), and we have recently reported that CXCL4 can induce myofibroblast differentiation in Gli1 ${ }^{+}$bone marrow cells (28). These data suggested that the circulating population might be involved in activation and myofibroblast differentiation of the resident population of myofibroblast precursors. We also checked the relative proliferative state of the 2 populations based on the scRNA-seq data indicating that both populations have a high percentage of cells in the $\mathrm{G}_{2} / \mathrm{M}$ and $\mathrm{S}$ phase of the cell cycle and, thus, are cells that show a proliferative response to kidney injury (Figure 6A) 
A

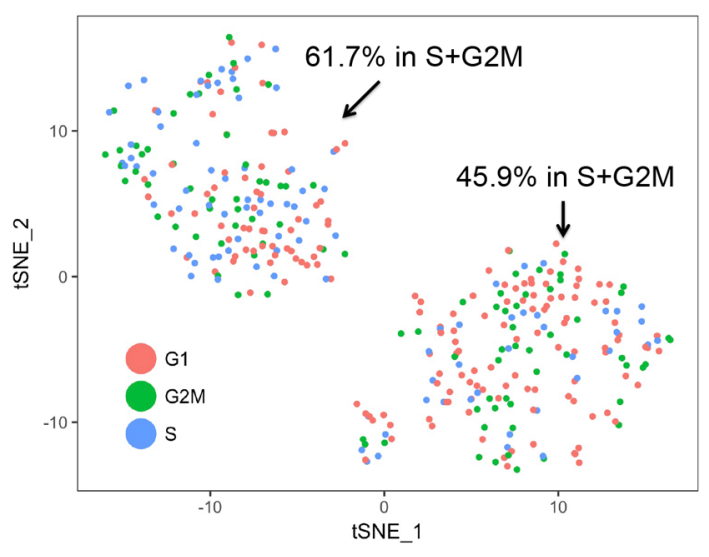

B

Cluster 1 - PDGFR $\beta^{+} / C D 45^{+}$
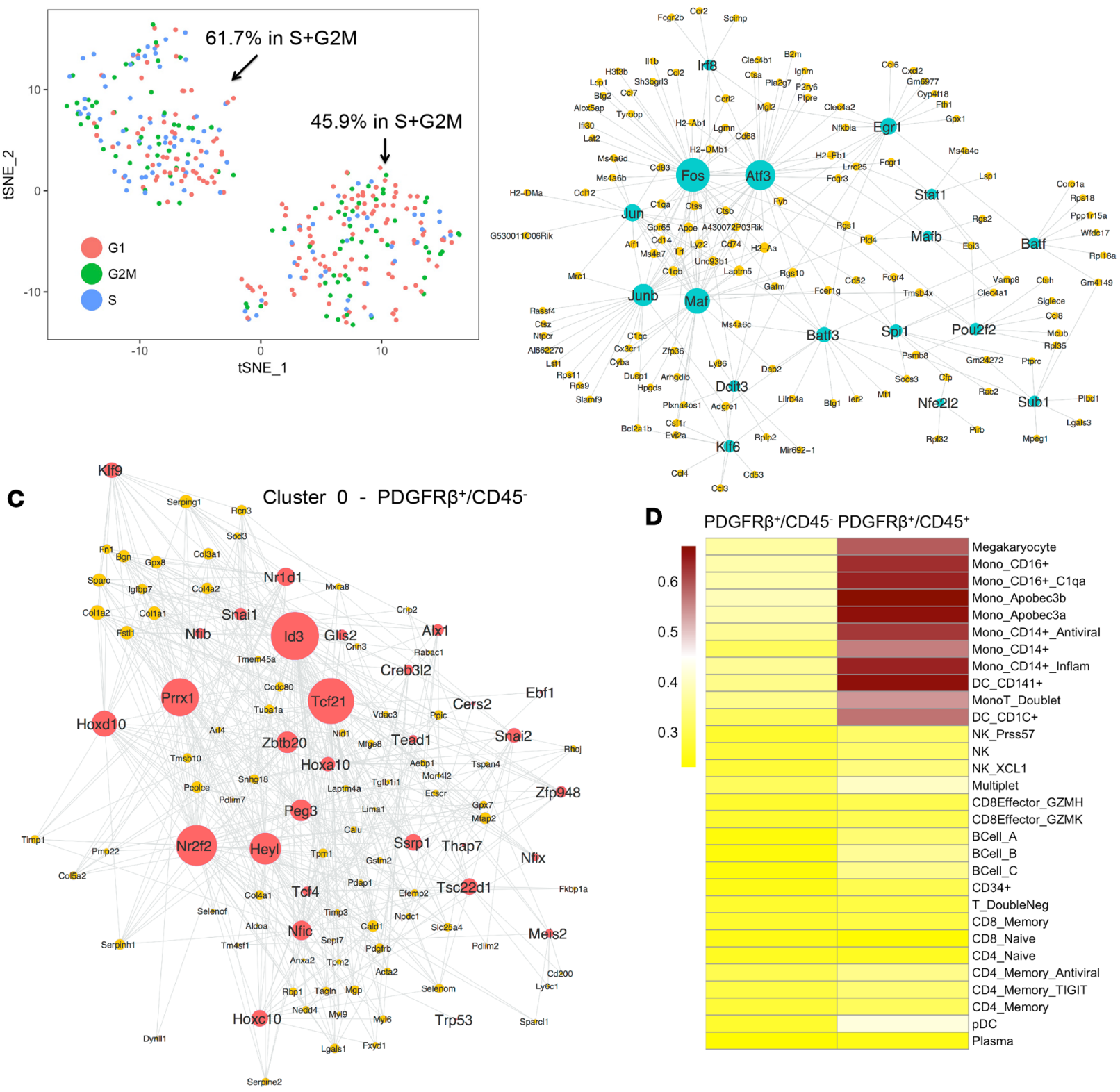

D $\quad \mathrm{PDGFR}^{+} / \mathrm{CD}^{-} 5^{-} \mathrm{PDGFR}^{+} / \mathrm{CD} 45^{+}$

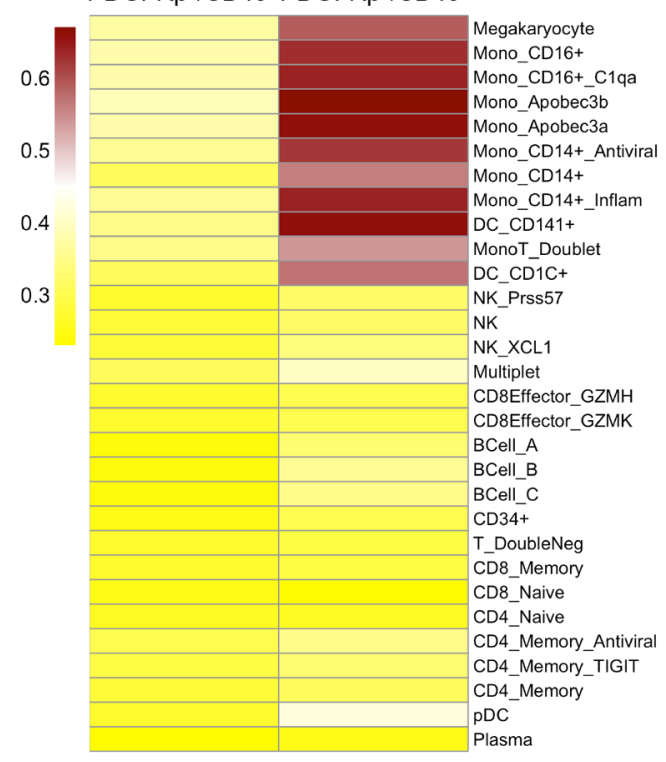

Figure 6. Single-cell RNA sequencing data identify circulating myofibroblast progenitors as monocytes. (A) Proliferative state in the 2 cell clusters based on gene expression. (B and C) Transcription factor (TF) activity network based on mRNA expression of TF target genes in the 2 cell populations. (D) Comparison of both cell populations with a Drop-Seq data set ( $10 \times$ genomics) of human peripheral blood mononuclear cells $(n=33,000$ cells, healthy donor). The color key denotes the Pearson's correlation coefficient (rho) between mouse and human cells. Rho has a value between -1 and 1 . A greater rho value indicates higher correlation between the two cell types.

The circulating myofibroblasts are of monocyte origin. Since transcription factors (TFs) drive cell identity, we next estimated TF activity based on their target gene (TG) expression in the scRNA-seq data. We aimed to identify key TFs that would define the 2 cell populations (Figure 6, B and C, and Supplemental Tables 1 and 2). The data showed 2 entirely distinct landscapes of TF activity in the 2 cell populations, further indicating that the 2 cell populations of resident $\left(\mathrm{PDGFR} \beta{ }^{+} \mathrm{CD} 45^{-}\right)$and circulating myofibroblasts $\left(\mathrm{PDGFR} \beta^{+} \mathrm{CD} 45^{+}\right)$are distinct from each other. In line with their expression of proinflammatory cytokines and their hematopoietic origin from circulation, the analysis revealed TFs that have been reported to be critical in the inflammatory response and macrophage activation, such as ATF3 (29), Fos (30), Jun/ Junb (31), Maf (32) and EGR1 (33) among others, were central in the landscape of TF activity of the PDGFR $\beta^{+} \mathrm{CD} 45^{+}$population (Figure 6, A and B, and Supplemental Table 1). 
In contrast, the resident population of myofibroblasts (PDGFR $\left.\beta^{+} \mathrm{CD} 45^{-}\right)$was characterized by TFs that have been reported to define tissue-resident cardiac fibroblasts/myofibroblasts, such as Tcf21 (34), or MSCs, such as Prrx1 or Hoxd10 (35-37). Furthermore, the data suggested high activity of the nuclear receptor subfamily 2 group member $2(\mathrm{Nr} 2 \mathrm{f} 2)$ in the resident PDGFR $\beta^{+} \mathrm{CD} 45^{-}$population. $\mathrm{Nr} 2 \mathrm{f} 2$ is an important inhibitor of adipogenesis and has been recently reported to be highly expressed in Gremlin $1^{+}$ bone marrow MSCs (38). These findings are in line with our previously reported fate-tracing experiments that found that kidney Gli1 ${ }^{+}$pericytes represent a population of MSCs and are a major source of myofibroblasts (4). We have further reported that Hedgehog/Gli signaling is a critical regulator of Gli1 ${ }^{+}$cell expansion and that inhibition of Gli proteins is a therapeutic strategy in kidney fibrosis (39). Consistent with these observations, the $\mathrm{Hh}$ pathway member and transcriptional repressor of $\mathrm{Hh}$ signaling, Glis2, was among other TFs enriched in the PDGFR $\beta^{+} \mathrm{CD} 45^{-}$population.

Gene ontology analysis revealed that genes involved in extracellular matrix formation, collagen production, metabolism, and growth factor binding were substantially overrepresented in the resident myofibroblast population $\left(\mathrm{PDGFR} \beta^{+} \mathrm{CD} 45^{-}\right.$), reflecting their major contribution to fibrosis formation (Supplemental Figure $3 \mathrm{~A}$ ). The circulating myofibroblast population of PDGFR $\beta^{+} \mathrm{CD} 45^{+}$cells mostly showed expression of genes that were associated with the immune response mechanism, reflecting their mechanism of activation and homing to the injured kidney (Supplemental Figure 3).

We next asked which circulating blood population might give rise to the small kidney PDGFR $\beta^{+} \mathrm{CD} 45^{+}$ myofibroblast population. We compared a published data set of human peripheral blood mononuclear cells (PBMCs) to the resident $\left(\mathrm{PDGFR} \beta^{+} \mathrm{CD} 45^{-}\right)$and circulating $\left(\mathrm{PDGFR} \beta^{+} \mathrm{CD} 45^{+}\right)$kidney myofibroblast data set (Figure 6D). Importantly, we did not observe any overlap of the PBMCs with the resident kidney myofibroblast population, whereas the circulating myofibroblast population of $\mathrm{PDGFR} \beta^{+} \mathrm{CD} 45^{+}$cells correlated strongly with human monocytes (Figure 6D). Altogether, these data indicate that circulating monocytes contribute a minor population of myofibroblasts; while they do not secrete much extracellular matrix, they produce high amounts of proinflammatory cytokines that might activate resident myofibroblasts. We next asked whether the circulating myofibroblast population of PDGFR $\beta^{+} \mathrm{CD} 45^{+}$cells shows expression of genes that have been reported to characterize M1 or M2 macrophages. Interestingly, PDGFR $\beta^{+} \mathrm{CD} 45^{+}$cells primarily showed expression of classically activated, proinflammatory M1 macrophages, such as Socs3, Tlr2, Il1b, TNFa, Ccl2, and Cc18 (Supplemental Figure 3B).

\section{Discussion}

Our data indicate that proximal tubular epithelium does not contribute to the myofibroblast pool, whereas circulating monocytes contribute a minor population of $\alpha \mathrm{SMA}^{+}$myofibroblasts. A contribution of monocytes and macrophages to the myofibroblast pool has been described by several groups $(18,23,24,40)$. However, Reich et al. have reported that fibrocytes independent of monocyte lineages are an important source of kidney myofibroblasts (17). As macrophages are a major site of collagen internalization and degradation, antibody staining for matrix proteins might be difficult to interpret (2). Importantly, there is an ongoing debate as to whether there is a direct contribution of myeloid leukocytes to the myofibroblast pool at all, and many reported effects might have been results of indirect mechanisms $(2,41)$. Indeed, in this work, we show, using two powerful and complementary approaches, that a small percentage of kidney myofibroblasts derive from monocytes. However, importantly, these myeloid-derived myofibroblasts express very few matrix genes - suggesting they do not play a direct role in interstitial matrix accumulation. The data indicate that PDGFR $\beta^{+} \mathrm{CD} 45^{+}$monocytes primarily secrete high amounts of proinflammatory profibrotic cytokines that have been reported to activate resident myofibroblasts, suggesting paracrine communications between circulating monocytes and resident myofibroblasts driving fibrosis as well. Thus, the direct contribution of circulating monocytes to the myofibroblast pool and matrix production is minor compared with their paracrine effects. This scRNA-seq data set confirms that the great majority of kidney myofibroblasts are of resident kidney origin.

A contribution of circulating bone marrow MSCs to the kidney myofibroblast pool has been discussed for several years as well (11). After parabiosis, two mice share one blood circulation, which allows rapid exchange of blood, cells, and particles, such as ultrasound bubbles, from one mouse to the other independent of cell size $(42,43)$. Therefore, our parabiosis approach, with induced labeling of all cells ( $90 \%$ recombination efficiency) in one mouse and induction of kidney fibrosis in the other mouse, allows quantitative tracking of all circulating cells, which would in theory also include MSCs. However, we found, through 
two independent experiments, that only $\mathrm{CD} 45^{+}$and thus hematopoietic (nonmesenchymal) cells contribute a small population of myofibroblast. Of note, CD45 expression is an exclusion criterion for MSCs.

First, the parabiosis experiments demonstrate that circulating tdTomato ${ }^{+}$cells in blood, spleen, and kidney are all $\mathrm{CD}_{4} 5^{+}$, and all of the circulating myofibroblasts we observed (tdTomato $\left.{ }^{+} / \alpha \mathrm{SMA}^{+}\right)$in the UUO kidneys also showed coexpression of CD45. However, in the parabiosis experiments, our recombination efficiency was $90 \%$, and, thus, although unlikely, we can not exclude that among the nonlabeled cells some nonhematopoietic cells might have contributed to the myofibroblast pool.

Second, since we have previously reported that all kidney myofibroblasts express PDGFR $\beta(4,13)$, we performed nonbiased scRNA-seq of PDGFR $\beta^{+}$cells that were either CD45 (hematopoietic) or CD45(nonhematopoietic) to profile all kidney myofibroblasts. The data indicate that the vast majority of kidney myofibroblasts are of resident origin, while a minor fraction of circulating hematopoietic $\left(\mathrm{CD} 45^{+}\right)$cells also expressed the myofibroblast marker $\alpha$ SMA. Importantly, when comparing the data set to a human PBMC scRNA-seq data set, only the circulating $\left(\mathrm{PDGFR} \beta^{+} \mathrm{CD} 45^{+}\right)$population showed a strong correlation, and the comparison identified monocytes as the origin of circulating myofibroblasts. Thus, our data indicate that circulating MSCs do not contribute to the myofibroblast pool.

Importantly, we have also recently reported bone marrow transplantation and parabiosis experiments, indicating that $\mathrm{Gli1}^{+} \mathrm{MSCs}$ do not circulate (4), and a recent study reports no evidence for circulating MSCs in patients with organ injury, unless they suffer severe trauma with multiple fractures, which leads to mechanical disruption of bone marrow with embolism (44). However, the scRNA-seq experiment was not performed as a time course, and thus we cannot account for dynamic changes in expression of the markers over the course of the experiment.

In conclusion, kidney myofibroblasts arise primarily from resident mesenchymal cells, whereas circulating monocytes contribute a minor fraction. Importantly, these circulating myofibroblasts primarily secrete proinflammatory cytokines and do not appreciably contribute to extracellular matrix production.

\section{Methods}

\section{Mice}

Rosa26CreER $^{\mathrm{t} 2}$ (JAX 008463), Rosa26tdTomato (JAX 007909), B6-CD45.1 (JAX 002014), and C57BL/6J (JAX 000664) mice were purchased from Jackson Laboratories. In the parabiosis experiment, bigenic Rosa26CreER;tdTomato mice (females, 8 weeks of age) received tamoxifen ( $4 \times 10 \mathrm{mg}$ in corn oil with $3 \%$ ethanol ) per oral gavage (every other day) and were conjoined with B6-CD45.1 mice (8-week-old females) 10 days after the last tamoxifen dose. Parabiosis was performed as previously described $(45,46)$.

Anesthesia was achieved by intraperitoneal injection of ketamine (100 mg/kg bodyweight), xylazine $(10 \mathrm{mg} / \mathrm{kg})$, and acepromazine $(3 \mathrm{mg} / \mathrm{kg})$. Buprenorphine $(0.05 \mathrm{mg} / \mathrm{kg})$, meloxicam $(1 \mathrm{mg} / \mathrm{kg})$, and lidocaine $(1 \%)$ were given subcutaneously to achieve analgesia.

The entire flank of each mouse was shaved, and the skin was cleaned with triple applications of a povidone-iodine soap, alternating with an alcohol rinse. Thereafter, matching skin incisions were made from the elbow to the knee joint of each mouse. The right olecranon of one animal was attached to the left olecranon of the other by a single 4-0 suture and tie. Similarly, the partners' knee joints were connected. The dorsal and ventral skin was then anastomosed by staples and suture. Four weeks after parabiosis, surgery blood was drawn from the retro-orbital vein plexus to check cross-circulation efficiency. Thereafter, the B6-CD45.1 mice underwent UUO surgery as follows. Anesthesia and analgesia was achieved in the parabiosis pair as described above. After flank incision, the right kidney was exposed and freed from the perirenal fat tissue and the ureter was tied off at the level of the lower pole using two 4.0 silk ties. Wounds were closed by staples. Mice were sacrificed at 10 days after UUO surgery. UUO surgeries in C57BL/6 wild-type mice were performed at 8 weeks of age using the same technique.

\section{Tissue preparation and histology}

Mice were anesthetized with isofluorane (Baxter) and subsequently perfused via the left ventricle with $4^{\circ} \mathrm{C}$ PBS for 1 minute. For histological analyses, tissue sections were fixed in $10 \%$ formaldehyde for 1 hour, paraffin embedded, cut with a rotating microtome at a thickness of $3 \mu \mathrm{m}$, and stained according to routine histological protocols. For immunofluorescence studies, kidneys were fixed in $4 \%$ paraformaldehyde on ice for 1 hour and then incubated in $30 \%$ sucrose in PBS at $4^{\circ} \mathrm{C}$ overnight. OCT-embedded (Sakura FineTek) tissues were 
cryosectioned into $5-\mu \mathrm{m}$ sections and mounted on Superfrost slides (Fisher Scientific). Sections were washed in $1 \times$ PBS, blocked in 10\% normal goat serum (Vector Labs), and incubated with primary antibodies specific for $\alpha$-SMA-FITC (mouse, 1:100, MilliporeSigma, catalog F3777), PDGFRb (rabbit, 1:100, Abcam, catalog ab32570), and CD45 (rat, 1:100, Novusbio, NB100-77417). Secondary antibodies were FITC- or Cy5-conjugated (Jackson ImmunoResearch). Sections were then stained with DAPI and mounted in Prolong Gold (Life Technologies). All images were obtained by confocal (Zeiss LSM880, equipped with an Airyscan detector) through the Washington University Center for Cellular Imaging. Fibrosis was scored at $\times 400$ magnification using a counting grid with 117 intersections. The number of grid intersections overlying trichrome-positive interstitial areas was counted and expressed as a percentage of all grid intersections. Intersections that were in tubular lumen and glomeruli were subtracted from the total number of grid intersections.

\section{Flow cytometry and cell sorting}

For flow cytometric analysis or FACS, mice were euthanized as described above and perfused with sterile PBS via the left ventricle, and the kidneys were placed in PBS with 5\% FBS. After thoroughly mincing the tissue/organ using a sterile scalpel (Feather), the tissue/organ was placed in gentleMACS C Tubes (Miltenyi Biotec) containing $1.5 \mathrm{ml}$ DMEM (Life Technologies) with $0.1 \mathrm{mg} / \mathrm{ml}$ Liberase TL (Roche). The tissue was then dissociated using the D program of the gentleMacs dissociator (Miltenyi Biotec) followed by 30 minutes incubation at $37^{\circ} \mathrm{C}$. Following washing steps with FACS buffer and centrifugation $(300 \mathrm{~g}$, 5 minutes), the solution was filtered twice through a $40-\mu \mathrm{m}$ cell strainer (BD Biosciences) and transfered to 5-ml Polystyrene Round-Bottom FACS tube (BD Biosciences). For flow cytometric studies, the samples were stained in 100-500 $\mu 1$ FACS buffer using the following fluorochrome-conjugated antibodies: CD45.1 (APC-Cy7, ebioscience, 25045382), CD45.2-FITC (ebioscience, 110454), $\alpha$-SMA-FITC (MilliporeSigma, F3777), PDGFRb-APC (Biolegend, 136008), and CD45-BV421 (Biolegend, 103133) all 1:100 for 30 minutes followed by a washing step with PBS. Cytofix/Cytoperm solution (BD Biosciences) was used to achieve staining for intracellular $\alpha$-SMA. All flow cytometric analyses were performed with a Canto II analyzer (BD Biosciences). For sorting, DAPI (1 mg/ml, 1:1,000) was added in order to exclude dead cells. Single-cell sorting was performed at the Siteman Flow Cytometry Core (Washington University in St. Louis) using an iCyt Synergy sorter (Sony). Single cells were sorted directly into $10 \times$ lysis buffer (Clontech) in 96-well PCR plates. Data were analyzed by using Flow Jo software (version 9.6.2, Tree Star Inc.).

\section{Single-cell RNA-sequencing and data preprocessing}

96-well plates with single cell in each well were sealed and sent to the sequencing core at Washington University in St. Louis (Genome Technology Access Center). RNA from individual wells was processed with the Clonetech Smarter system and ligated with unique barcodes. Each plate was then pooled into one library, and the resulting pools were then ligated to adapters containing unique 7-bp index sequences, so that samples originating from a single plate can be identified by Illumina conventional indexing strategies and each individual well is defined by barcodes sequenced on the first read of a paired end read pair.

All plates were pooled into a single library and subjected to Illumina sequencing (HiSeq2500, $2 \times 50 \mathrm{bp}$ ). The resulting sequencing reads were demultiplexed by index with a custom Phython demultiplexing script and further demultiplexed by barcode with a custom Perl demultiplexing script. The RNA-sequencing reads were then aligned to the Mus musculus Ensembl release 76 top-level assembly with STAR version 3.0.4.b. Gene counts were derived from the number of uniquely aligned unambiguous reads by Subread:featureCount version 1.4.5. Sailfish version 0.6.3 was used to produce isoform estimated counts. Gene and isoform counts were further transformed into counts per million (CPM), using log2 CPM with a prior count of 2 (moderated $\log _{2} \mathrm{CPM}$ ), and RPKM with custom Rscripts. Sequencing performance was assessed for the total number of aligned reads, the total number of uniquely aligned reads, the genes and transcripts detected, the ribosomal fraction, the known junction saturation, and the read distribution over known gene models with $\mathrm{RSeQC}$ version 2.3. The single-cell RNA-sequencing raw data are available in the Gene Expression Omnibus database (accession GSE112033).

\section{Bioinformatics}

Unsupervised clustering and differential gene analysis. We used Seurat software in R to identify cell clusters, performed differential gene analysis, and generated the top gene heatmap. In brief, a gene expression matrix with gene names as rows and cell names as columns was input into Seurat. Before clustering, we removed 
genes with low expression levels and low-quality cells by filtering out genes that were expressed in less than 10 cells and cells that had a greater than $20 \%$ mitochondrial gene content, resulting in 14,062 genes and 357 cells. After filtering, we normalized the expression values for each cell by the total expression, multiplied this by 10,000 , and log transformed the result. Next, we performed dimensionality reduction on the scaled data by computing the significant principal components (PCs) on highly variable genes. Graph-based clustering analyses were performed on the first 10 PCs, and clusters were visualized in tSNE. To examine potential batch effect, cells were colored by plates and plotted in the same tSNE. Differential gene analysis was performed using the FindAllMarkers function in Seurat (likelihood-ratio test). Differentially expressed genes that were expressed at least in $25 \%$ cells within the cluster and with a fold change of more than 0.25 (log scale) were considered to be marker genes. Marker genes in each cluster were visualized by two different ways: we used the DoHeatmap function (Seurat) to show the top 30 genes for each cluster and the FeaturePlot function (Seurat) to plot the well-known markers. To compare the relative expression of profibrotic genes and proinflammatory genes between clusters, we selected genes that are components of extracellular matrix and genes that are known as cytokines/chemokines from the differential gene list. The average expression level of these selected genes was $Z$-score normalized and visualized using the pheatmap package in $\mathrm{R}$. To classify the gene function of the marker genes, we uploaded the differential gene list onto ToppGene suite (https://toppgene.cchmc.org) for gene set functional enrichment analysis. The top 5 enriched gene ontology terms from each category (molecular function, cellular components, and biological function) were compared among clusters, and bar graphs shown in figures were generated using the ggplot2 package.

Comparison of fibrocytes and a public PBMC data set. Cell-type-specific expression patterns of the cell clusters identified in our data set were compared with signatures previously defined in a PBMC data set by calculating the pairwise Pearson correlations coefficients between each pair of cell types for the same set of genes. First, a precomputed Seurat object containing cell cluster information for 33,000 human PBMCs was downloaded from Satija Lab (http://satijalab.org/seurat/get_started.html). Second, mouse genes were converted to human equivalent genes using a $\mathrm{R}$ package biomaRt. Only genes that are detected in our data set and the PBMC data set were used for downstream correlation analysis. Third, Pearson correlation was computed between the cell clusters in our data set and the cell clusters identified in the PBMC data set, using the previously defined cell-type annotations and normalized average gene expression values for each cell type. Data were shown by pheatmap R package.

Construction of transcription network by driving force analysis. To identify the key regulators that control the cell states, we established the relationship between TFs and their TGs following the SINCERA pipeline (47). This approach consists of three main steps. First, the candidate TFs and TGs were extracted from the DEG list identified in each cluster. Second, cell-type-specific transcription regulatory network (TRN) was constructed by establishing the interaction between TF-TF and TF-TG as previously described (48). TG-TF and TF autoregulations were not considered in the present study. Finally, the key TFs were selected based on their network node importance and ranked by integrating 6 node importance metrics, including degree centrality, closeness centrality, betweenness centrality, disruptive fragmentation centrality, disruptive connection centrality, and disruptive distance centrality. The TRN igraph object obtained from SINCERA was converted to JSON using a R package RJSONIO and imported into Cytoscape (version 3.4.0) for network visualization.

\section{Ligand-receptor interaction analysis}

To study ligand-receptor interactions between myofibroblast and fibrocyte, we used a human ligand-receptor list comprising 2,557 ligand-receptor pairs curated by the Database of Ligand-Recetor Partners, IUPHAR, and Human Plasma Membrane Receptome (49). We selected the receptors that were only differentially expressed in each cell type. To determine the ligand-receptor pairs to plot on the heatmap, we required that (a) the receptors are uniquely expressed in each cell type $(q<0.05$ and log fold change $>0.6)$ and (b) each receptor should have at least one corresponding ligand to pair with. We used heatmap.2 function from gplots package to visualize the ligand-receptor pairs.

\section{Statistics}

Data are presented as mean \pm SD. Comparison of two groups was performed using unpaired 2-tailed $t$ test. Paired 2-tailed $t$ test was used for comparison of repeated measured in the same group. Statistical analyses were performed using GraphPad Prism 5.0c (GraphPad Software Inc.). A $P$ value of less than 0.05 was considered significant. 


\section{Study approval}

All mouse experiments were performed with the approval of and according to the animal experimental guidelines issued by the Animal Care and Use Committee at Harvard University, Boston, Massachusetts, USA (\#04474), and the Washington University in St. Louis, St. Louis, Missouri, USA (\#A-3381-01).

\section{Author contributions}

RK designed and carried out experiments, analyzed results, and wrote the manuscript. FM, KH, TK, and RKS carried out some of the experiments, analyzed the data, and reviewed the manuscript. HW contributed to the scRNA-seq experiment and performed the bioinformatics. BDH designed experiments, analyzed results, and contributed to the writing of the manuscript.

\section{Acknowledgments}

This work was supported by grants from the German Research Foundation (KR-4073/3-1, SCHN1188/5-1, SFB/TRR57, and SFB/TRR219), a grant from the European Research Council (ERC-StG 677448), a START grant from RWTH Aachen University (101/15), a grant from the State of North Rhine Westphalia (Return to NRW) to RK, grants from the NIH/National Institute of Diabetes and Digestive and Kidney Diseases (DK088923, DK103740, and DK103050), and by an Established Investigator Award from the American Heart Association (EIA14650059) to BDH. We thank the Genome Technology Access Center in the Department of Genetics at Washington University School of Medicine for help with genomic analysis. The Center is partially supported by National Cancer Institute Cancer Center support grant P30 CA91842 to the Siteman Cancer Center and by the Institute for Clinical and Translational Science/Clinical and Translational Sciences Award grant UL1 TR000448 from the National Center for Research Resources (NCRR), a component of the NIH and NIH Roadmap for Medial Research. This publication is solely the responsibility of the authors and does not necessarily represent the official view of the NCRR or NIH. Experiments were performed in part through the use of Washington University Center for Cellular Imaging.

Address correspondence to: Rafael Kramann, Division of Nephrology and Clinical Immunology, Medical Faculty, RWTH Aachen University, Pauwelsstrasse 30, 52074 Aachen, Germany. Phone: 0049.241.80.37750; Email: rkramann@gmx.net. Or to: Benjamin D. Humphreys, Division of Nephrology, Washington University School of Medicine, 660 S. Euclid Avenue, CB8126, St. Louis, Missouri 63110, USA. Phone: 314.362.8233; Email: humphreysbd@wustl.edu.

1. El Agha E, et al. Mesenchymal stem cells in fibrotic disease. Cell Stem Cell. 2017;21(2):166-177.

2. Duffield JS. Cellular and molecular mechanisms in kidney fibrosis. J Clin Invest. 2014;124(6):2299-2306.

3. Falke LL, Gholizadeh S, Goldschmeding R, Kok RJ, Nguyen TQ. Diverse origins of the myofibroblast-implications for kidney fibrosis. Nat Rev Nephrol. 2015;11(4):233-244.

4. Kramann R, et al. Perivascular Gli1+ progenitors are key contributors to injury-induced organ fibrosis. Cell Stem Cell. 2015;16(1):51-66.

5. Kriz W, Kaissling B, Le Hir M. Epithelial-mesenchymal transition (EMT) in kidney fibrosis: fact or fantasy? J Clin Invest. 2011;121(2):468-474.

6. Menon MC, Ross MJ. Epithelial-to-mesenchymal transition of tubular epithelial cells in renal fibrosis: a new twist on an old tale. Kidney Int. 2016;89(2):263-266.

7. Zhao Y, et al. MiR-30c protects diabetic nephropathy by suppressing epithelial-to-mesenchymal transition in db/db mice. Aging Cell. 2017;16(2):387-400.

8. Grande MT, et al. Snaill-induced partial epithelial-to-mesenchymal transition drives renal fibrosis in mice and can be targeted to reverse established disease. Nat Med. 2015;21(9):989-997.

9. Humphreys BD, et al. Fate tracing reveals the pericyte and not epithelial origin of myofibroblasts in kidney fibrosis. Am J Pathol. 2010;176(1):85-97.

10. Iwano M, Plieth D, Danoff TM, Xue C, Okada H, Neilson EG. Evidence that fibroblasts derive from epithelium during tissue fibrosis. J Clin Invest. 2002;110(3):341-350.

11. LeBleu VS, et al. Origin and function of myofibroblasts in kidney fibrosis. Nat Med. 2013;19(8):1047-1053.

12. Kramann R, Dirocco DP, Maarouf OH, Humphreys BD. Matrix producing cells in chronic kidney disease: origin, regulation, and activation. Curr Pathobiol Rep. 2013;1(4).

13. Kramann R, DiRocco DP, Humphreys BD. Understanding the origin, activation and regulation of matrix-producing myofibroblasts for treatment of fibrotic disease. J Pathol. 2013;231(3):273-289.

14. Bucala R, Spiegel LA, Chesney J, Hogan M, Cerami A. Circulating fibrocytes define a new leukocyte subpopulation that mediates tissue repair. Mol Med. 1994;1(1):71-81.

15. Roufosse C, et al. Bone marrow-derived cells do not contribute significantly to collagen I synthesis in a murine model of renal 
fibrosis. J Am Soc Nephrol. 2006;17(3):775-782.

16. Lin SL, Kisseleva T, Brenner DA, Duffield JS. Pericytes and perivascular fibroblasts are the primary source of collagen-producing cells in obstructive fibrosis of the kidney. Am J Pathol. 2008;173(6):1617-1627.

17. Reich B, et al. Fibrocytes develop outside the kidney but contribute to renal fibrosis in a mouse model. Kidney Int. 2013;84(1):78-89.

18. Wang YY, et al. Macrophage-to-myofibroblast transition contributes to interstitial fibrosis in chronic renal allograft injury. $J A m$ Soc Nephrol. 2017;28(7):2053-2067.

19. Broekema M, et al. Bone marrow-derived myofibroblasts contribute to the renal interstitial myofibroblast population and produce procollagen I after ischemia/reperfusion in rats. J Am Soc Nephrol. 2007;18(1):165-175.

20. Chen G, et al. CXCL16 recruits bone marrow-derived fibroblast precursors in renal fibrosis. J Am Soc Nephrol. 2011;22(10):1876-1886.

21. Yan J, Zhang Z, Yang J, Mitch WE, Wang Y. JAK3/STAT6 stimulates bone marrow-derived fibroblast activation in renal fibrosis. J Am Soc Nephrol. 2015;26(12):3060-3071.

22. Li J, Deane JA, Campanale NV, Bertram JF, Ricardo SD. The contribution of bone marrow-derived cells to the development of renal interstitial fibrosis. Stem Cells. 2007;25(3):697-706.

23. Yang J, et al. Adiponectin promotes monocyte-to-fibroblast transition in renal fibrosis. J Am Soc Nephrol. 2013;24(10):1644-1659.

24. Wang $S$, et al. TGF- $\beta / \operatorname{Smad} 3$ signalling regulates the transition of bone marrow-derived macrophages into myofibroblasts during tissue fibrosis. Oncotarget. 2016;7(8):8809-8822.

25. Cilloni D, et al. Limited engraftment capacity of bone marrow-derived mesenchymal cells following T-cell-depleted hematopoietic stem cell transplantation. Blood. 2000;96(10):3637-3643.

26. Kusaba T, Lalli M, Kramann R, Kobayashi A, Humphreys BD. Differentiated kidney epithelial cells repair injured proximal tubule. Proc Natl Acad Sci USA. 2014;111(4):1527-1532.

27. Aono Y, et al. Role of platelet-derived growth factor/platelet-derived growth factor receptor axis in the trafficking of circulating fibrocytes in pulmonary fibrosis. Am J Respir Cell Mol Biol. 2014;51(6):793-801.

28. Schneider RK, et al. Gli1+ mesenchymal stromal cells are a key driver of bone marrow fibrosis and an important cellular therapeutic target. Cell Stem Cell. 2017;20(6):785-800.e8.

29. Thompson MR, Xu D, Williams BR. ATF3 transcription factor and its emerging roles in immunity and cancer. $J M o l ~ M e d$. 2009;87(11):1053-1060.

30. Zenz R, et al. Activator protein 1 (Fos/Jun) functions in inflammatory bone and skin disease. Arthritis Res Ther. $2008 ; 10(1): 201$.

31. Fontana MF, Baccarella A, Pancholi N, Pufall MA, Herbert DR, Kim CC. JUNB is a key transcriptional modulator of macrophage activation. J Immunol. 2015;194(1):177-186.

32. Cao S, Liu J, Song L, Ma X. The protooncogene c-Maf is an essential transcription factor for IL-10 gene expression in macrophages. J Immunol. 2005;174(6):3484-3492.

33. Decker EL, Skerka C, Zipfel PF. The early growth response protein (EGR-1) regulates interleukin-2 transcription by synergistic interaction with the nuclear factor of activated T cells. J Biol Chem. 1998;273(41):26923-26930.

34. Kanisicak O, et al. Genetic lineage tracing defines myofibroblast origin and function in the injured heart. Nat Commun. 2016;7:12260.

35. Kumar A, et al. Specification and diversification of pericytes and smooth muscle cells from mesenchymoangioblasts. Cell Rep. 2017;19(9):1902-1916.

36. Greenbaum A, et al. CXCL12 in early mesenchymal progenitors is required for haematopoietic stem-cell maintenance. Nature 2013;495(7440):227-230.

37. Ackema KB, Charité J. Mesenchymal stem cells from different organs are characterized by distinct topographic Hox codes. Stem Cells Dev. 2008;17(5):979-991.

38. Worthley DL, et al. Gremlin 1 identifies a skeletal stem cell with bone, cartilage, and reticular stromal potential. Cell. 2015;160(1-2):269-284.

39. Kramann R, et al. Pharmacological GLI2 inhibition prevents myofibroblast cell-cycle progression and reduces kidney fibrosis. J Clin Invest. 2015;125(8):2935-2951.

40. Nikolic-Paterson DJ, Wang S, Lan HY. Macrophages promote renal fibrosis through direct and indirect mechanisms. Kidney Int Suppl (2011). 2014;4(1):34-38.

41. Nelson PJ. The contentious ontogeny of fibrosis in the kidney. Kidney Int. 2013;84(1):14-15.

42. Gibney BC, et al. Cross-circulation and cell distribution kinetics in parabiotic mice. J Cell Physiol. 2012;227(2):821-828.

43. Wu JM, et al. Circulating cells contribute to cardiomyocyte regeneration after injury. Circ Res. 2015;116(4):633-641.

44. Hoogduijn MJ, et al. No evidence for circulating mesenchymal stem cells in patients with organ injury. Stem Cells Dev. 2014;23(19):2328-2335.

45. Bunster E, Meyer RK. An improved method of parabiosis. Anat Rec. 1933;57:339-380.

46. Ruckh JM, et al. Rejuvenation of regeneration in the aging central nervous system. Cell Stem Cell. 2012;10(1):96-103.

47. Guo M, Wang H, Potter SS, Whitsett JA, Xu Y. SINCERA: A pipeline for single-cell RNA-Seq profiling analysis. PLoS Comput Biol. 2015;11(11):e1004575.

48. Lèbre S. Inferring dynamic genetic networks with low order independencies. Stat Appl Genet Mol Biol. 2009;8:Article 9

49. Ramilowski JA, et al. A draft network of ligand-receptor-mediated multicellular signalling in human. Nat Commun. $2015 ; 6: 7866$. 